\title{
Guidance on the Measurement of Temporal Light Modulation of Light Sources and Lighting Systems
}

Technical note

Schakel, M.; Banerjee, K.; Bergen, T.; Blattner, P.; Bouroussis, C.; Dekker, P.; Klej, A.; Li, C.; Ootake, H.; Reiners, T.

Total number of authors:

13

Link to article, DOI:

$10.25039 /$ TN.012.2021

Publication date:

2021

Document Version

Publisher's PDF, also known as Version of record

Link back to DTU Orbit

Citation (APA):

Schakel, M., Banerjee, K., Bergen, T., Blattner, P., Bouroussis, C., Dekker, P., Klej, A., Li, C., Ootake, H., Reiners, T., Sperling, A., Thorseth, A., \& Zuber, R. (2021). Guidance on the Measurement of Temporal Light Modulation of Light Sources and Lighting Systems: Technical note. CIE - International Commission on Illumination. CIE Technical Notes Vol. 2021 No. 012 https://doi.org/10.25039/TN.012.2021

\section{General rights}

Copyright and moral rights for the publications made accessible in the public portal are retained by the authors and/or other copyright owners and it is a condition of accessing publications that users recognise and abide by the legal requirements associated with these rights.

- Users may download and print one copy of any publication from the public portal for the purpose of private study or research.

- You may not further distribute the material or use it for any profit-making activity or commercial gain

- You may freely distribute the URL identifying the publication in the public portal 
Commission Internationale de l'Eclairage

Internationale Beleuchtungskommission
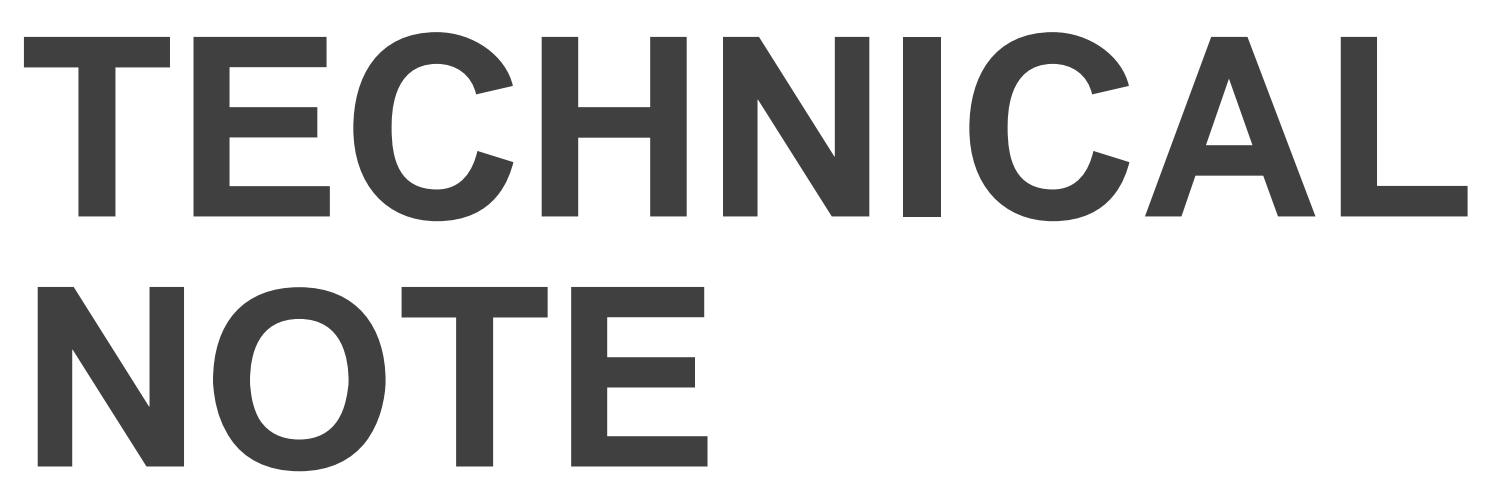

\section{Guidance on the Measurement of Temporal Light Modulation of Light Sources and Lighting Systems}


CIE Technical Notes (TN) are concise technical papers summarizing information of fundamental importance to CIE Members and other stakeholders, which either have been prepared by a TC, in which case they will usually form only a part of the outputs from that TC, or through the auspices of a Reportership established for the purpose in response to a need identified by a Division or Divisions.

This Technical Note has been prepared by CIE TC 2-89 of Division 2 "Physical Measurement of Light and Radiation". It has been approved by the Board of Administration and Division 2 of $\mathrm{CIE}$. The document reports on current knowledge and experience within the specific field of light and lighting described, and is intended to be used by the CIE membership and other interested parties. It should be noted, however, that the status of this document is advisory and not mandatory.

Any mention of organizations or products does not imply endorsement by the CIE. Whilst every care has been taken in the compilation of any lists, up to the time of going to press, these may not be comprehensive.

(C) CIE 2021 - All rights reserved 
The following members of TC 2-89 "Measurement of Temporal Light Modulation of Light Sources and Lighting Systems" took part in the preparation of this Technical Note. The committee comes under Division 2 "Physical measurement of light and radiation".

Authors:

Schakel, M. (Chair)

Banerjee, K.

Bergen, $T$.

Blattner, $P$

Bouroussis, C.

Dekker, P.

Klej, A.

Li, C

Ootake, $\mathrm{H}$.

Reiners, $\mathrm{T}$.

Sperling, A.

Thorseth, A.

Zuber, R.

\section{Germany}

Netherlands

Australia

Switzerland

Greece

Netherlands

Netherlands

China

Japan

Germany

Germany

Denmark

Germany 


\section{CONTENTS}

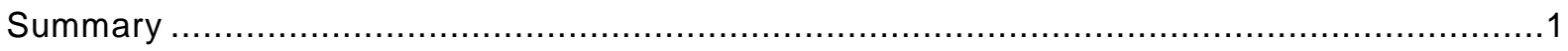

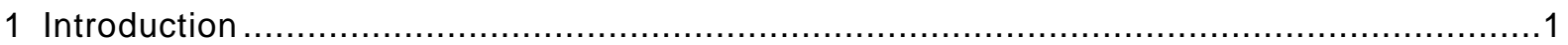

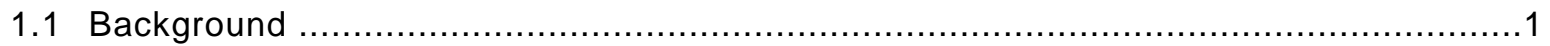

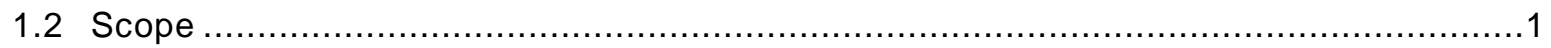

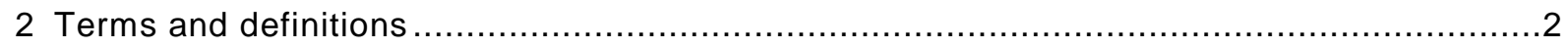

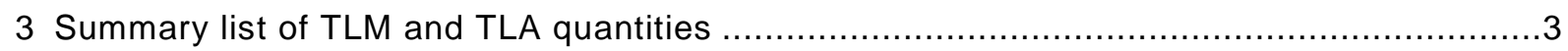

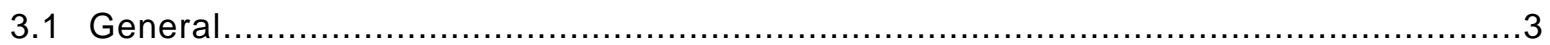

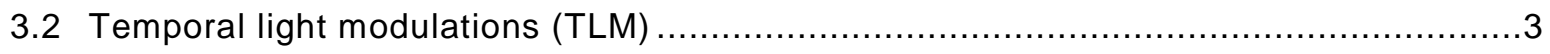

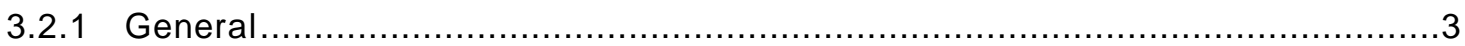

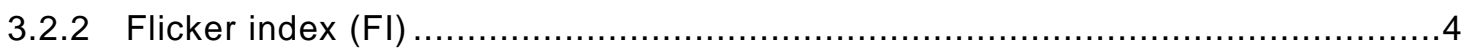

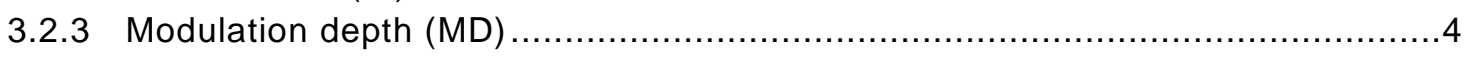

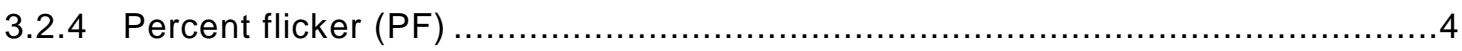

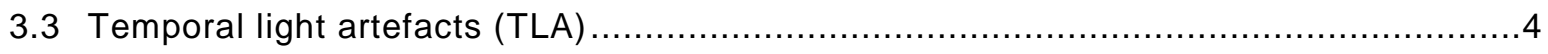

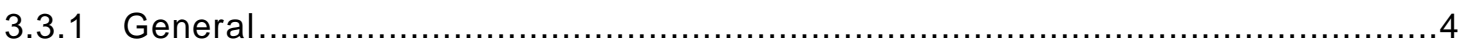

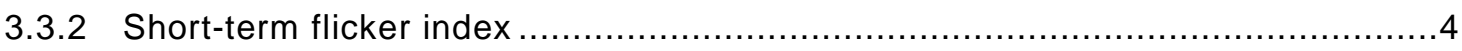

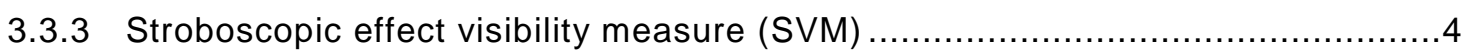

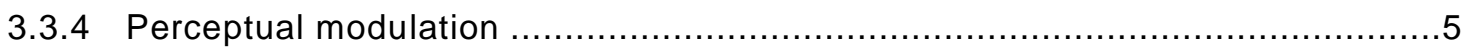

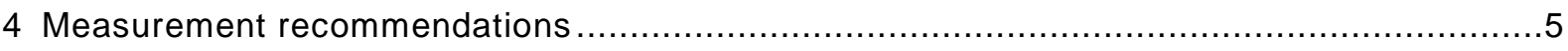

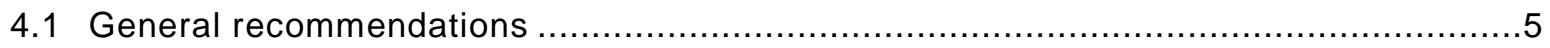

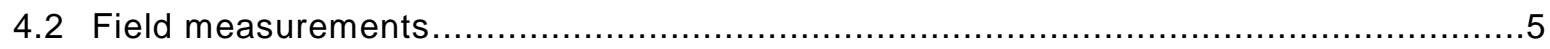

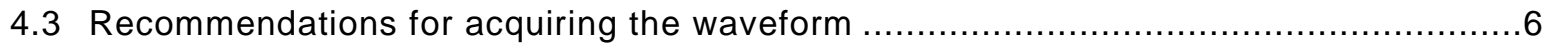

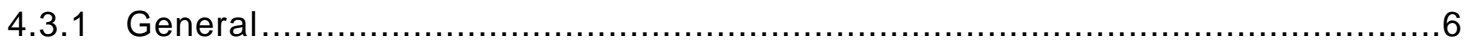

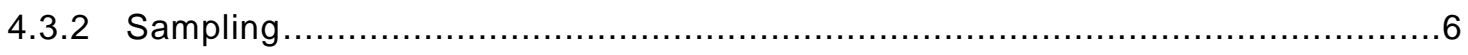

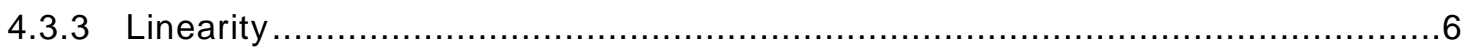

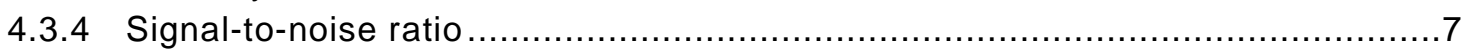

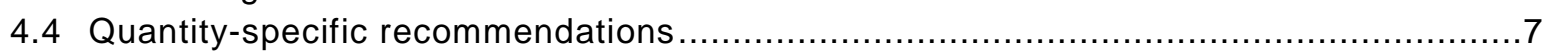

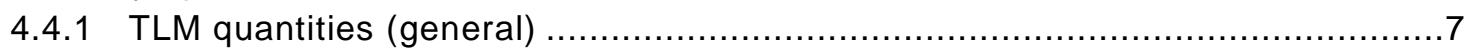

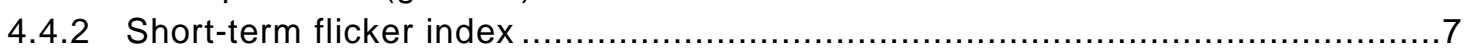

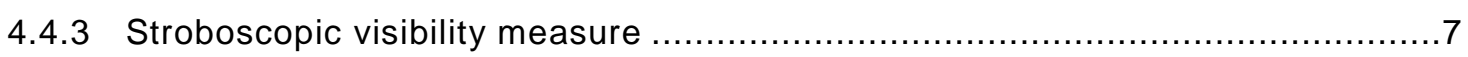

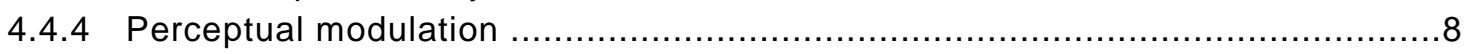

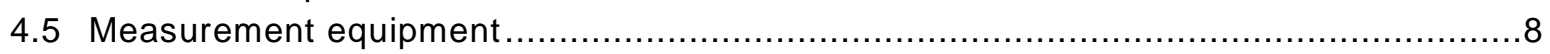

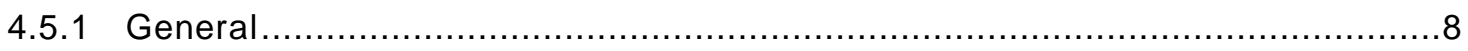

4.5.2 Measured quantities and measurement geometries .................................. 8

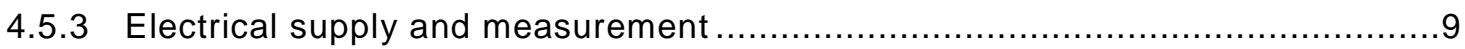

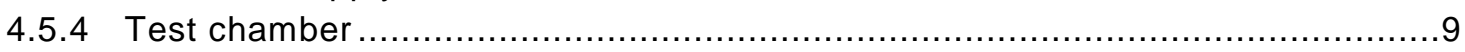

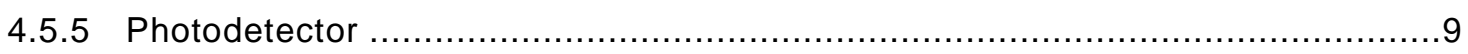

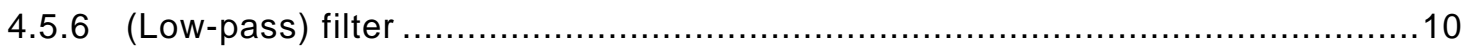

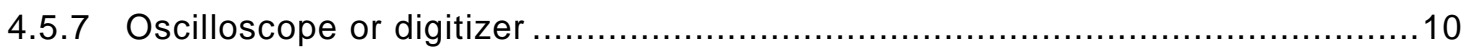

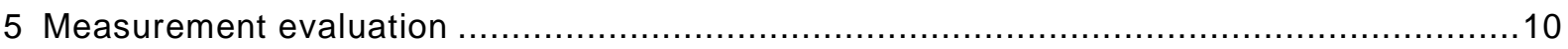

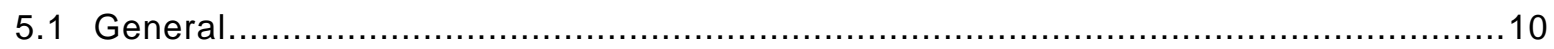

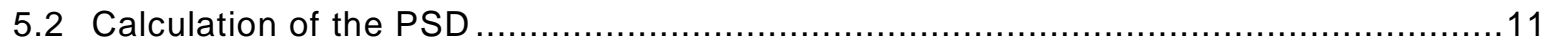

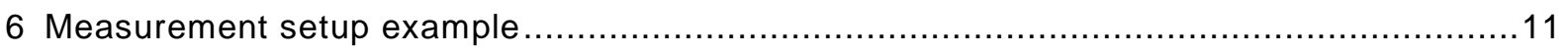

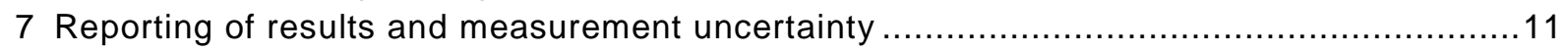

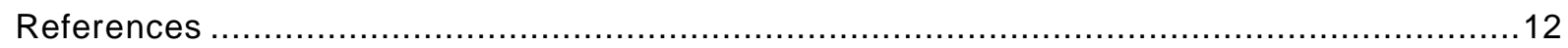




\section{Summary}

New regulations are coming into force in several regions with respect to temporal light modulation of lighting products. However, standardized test methods and even basic understanding of requirements are largely lacking in the area. Newly introduced metrics, like the stroboscopic visibility measure, are used in these regulations without the existence of standardized measurement methods.

This document sets the stage for an understanding of these new metrics and provides guidelines for the correct measurement of them. The document at hand is meant to provide respective recommendations, which do not imply any kind of standardization.

\section{Introduction}

\section{$1.1 \quad$ Background}

In 2016 CIE TN 006 "Visual Aspects of Time-Modulated Lighting Systems - Definitions and Measurement Models" (CIE 2016) was published. CIE TN 006 describes measurement principles to characterize the visibility of temporal light artefacts (TLA) of light sources.

The term "temporal light modulation" (TLM) is used for any measurable change of the light level or the spectral distribution of light over time. The term "temporal light artefacts" (TLA) is only used for the human visual perception of these modulations. The most important reason to have a measurement method for TLM is that exposure to light modulation may cause undesired effects on human perception (TLA), health, performance and safety.

After the introduction of LEDs to lighting products, the measurement of light modulation has become more and more important. Although LEDs do not intrinsically produce TLM, they do have a fast reaction time to the driving current. This means that any associated waveform, whether intentional or unintentional, changes from the electrical input (e.g. control gear or direct mains) can also result in a change in the output of the LEDs and the perception of these changes.

Currently there is a wide variety of quantities for TLM and TLA. The most commonly used ones are summarized in Clause 3.

\subsection{Scope}

This document provides recommendations on measurement protocols to measure periodic waveforms and light modulations. The recommendations should enable measurement laboratories to apply the same measurement methodology and to report the results in a consistent and reproducible way.

This document covers methods of measurement for TLM and TLA of lighting equipment. Its primary application is for general lighting purposes; however, the principles can be applied to other fields (e.g. display equipment, or facade lighting), though these generally require different input optics for the measurement equipment.

The recommendations given in this document can be used to measure non-periodic signals, but there might be specific aspects of these signals that will not be covered in this document (e.g. signal-triggering). 


\section{Terms and definitions}

\section{1}

\section{temporal light modulation}

TLM

fluctuation in luminous quantity or spectral distribution of light with respect to time

\section{2}

\section{temporal light artefact}

\section{TLA}

change in visual perception, induced by a light stimulus, the luminance or spectral distribution of which fluctuates with time, for a human observer in a specified environment

Note 1 to entry: The change of visual perception is a result of comparing the visual perception of the environment lit by the modulated light to the visual perception of the same person in the same environment, when the environment is lit by non-modulated light.

[Source: CIE TN 006:2016, 2.4.1]

\section{3}

flicker

perception of visual unsteadiness induced by a light stimulus the luminance or spectral distribution of which fluctuates with time, for a static observer in a static environment

Note 1 to entry: The fluctuations of the light stimulus with time include periodic and non-periodic fluctuations and may be induced by the light source itself, the power source or other influencing factors.

Note 2 to entry: Flicker is a type of temporal light artefact.

[SOURCE: CIE S 017:2020, Entry 17-22-092, modified - Note 2 to entry added]

\section{4}

\section{stroboscopic effect}

change in motion perception induced by a light stimulus the luminance or spectral distribution of which fluctuates with time, for a static observer in a non-static environment

Note 1 to entry: The stroboscopic effect is a type of temporal light artefact.

EXAMPLE 1 For a square periodic luminance fluctuation, moving objects are perceived to move discretely rather than continuously.

EXAMPLE 2 If the frequency of a periodic luminance fluctuation coincides with the frequency of a rotating object, the rotating object is perceived as static.

[Source: CIE TN 006:2016, 2.4.3]

\section{5}

\section{waveform}

representation of a characteristic quantity of a wave, either in time at a given point or in space at a given time

Note 1 to entry: If the quantity in question is a luminous quantity (e.g. luminance, luminous intensity, or illuminance), this is called a "light waveform".

[Source: IEC 60050-103:2009, Entry 103-10-02, modified - Note 1 to entry added] 


\section{6}

\section{modulation}

process by which a quantity which characterizes an oscillation or wave follows the variations of a signal or of another oscillation or wave

Note 1 to entry: Modulation may be intentional or unintentional.

[Source: IEC 60050-701:1988, Entry 701-03-08, modified - "." at the end of the definition removed]

\section{7}

steady state, <of a light source>

state of operation of a light source, which has been stabilized for a sufficient period of time such that the root-mean-square (RMS) and mean values of the electrical and photometric quantities become stable

\section{8}

period, <of a signal>

smallest positive difference between two values of the independent variable at which the values of a periodic quantity are identically repeated

[Source: IEC 60050-103:2009, Entry 103-06-01, modified - specifier added and notes to entry omitted]

\section{9}

dominant frequency, <of a periodic signal>

non-zero frequency component with the highest energy content

\subsection{0}

\section{power spectral density}

\section{PSD}

distribution as a function of frequency of the power per unit bandwidth of the spectral components of a signal or a noise having a continuous spectrum and a finite mean power

Note 1 to entry: One way of calculating the PSD is by Fourier Transform of the waveform signal.

[Source: IEC 60050-713:1998, Entry 713-09-12, Note 1 to entry added]

\section{Summary list of TLM and TLA quantities}

\subsection{General}

The list below is not an exhaustive list. It is accumulated from several sources and is only listed for reference. References are given where available.

\subsection{Temporal light modulations (TLM)}

\subsubsection{General}

The quantities that are classified as temporal light modulations quantify the amount or level of modulation. These quantities do not have a direct connection to the visual perception of temporal light artefacts. Generally, they are measured over one cycle (or averaged over several cycles) of a repeating waveform (e.g. square, sine) under steady state conditions. 


\subsubsection{Flicker index (FI)}

According to CIE S 017:2020, 17-22-094 (CIE 2020) the flicker index (symbol IF) is the quotient of the above-average luminous energy to the total luminous energy over a period of time.

\subsubsection{Modulation depth (MD)}

The modulation depth (symbol $D \mathrm{M}$ ) is defined as

$$
D_{\mathrm{M}}=\frac{y_{\max }-y_{\min }}{y_{\max }+y_{\min }}
$$

where $y_{\max }$ is the maximum signal, and $y_{\min }$ is the minimum signal of the waveform within a period of time.

\subsubsection{Percent flicker (PF)}

Percent flicker is a relative measure of the maximum value of a waveform compared to the minimum value. The calculation is the same as for modulation depth but is presented as a percentage. It is therefore often used as a synonym for modulation depth.

\subsection{Temporal light artefacts (TLA)}

\subsubsection{General}

The quantities that are classified as temporal light artefacts have a direct relation to the human perception of light modulation.

Generally, these quantities are measured over a longer section of the waveform and are not restricted to a repetitive waveform, i.e. complex signals can also be analysed.

\subsubsection{Short-term flicker index}

As a measure of the short-term fluctuations of the light output of a device that the mains voltage supply may produce, the International Electrotechnical Commission (IEC) developed the flickermeter method. It predicts flicker visibility of an incandescent lamp connected to the electrical power system caused by rapid voltage fluctuations in the systems. The method consists of a few components or "blocks", one of which simulates the human visual response to flickering light. One of the components of the flickermeter uses a model of a $60 \mathrm{~W}$ incandescent lamp as a reference light source that translates the voltage fluctuations on the mains to visible radiation fluctuations. This block can be removed, and the measured light output can be directly fed to the rest of the processing blocks, resulting in the light flickermeter (IEC, 2017).

The output of this flickermeter is the short-term flicker index (symbol $P_{\mathrm{st}}^{\mathrm{LM}}$ ) in which the wave shape and frequency are taken into consideration since the measurement is carried out over a longer time (generally more than one minute).

\subsubsection{Stroboscopic effect visibility measure (SVM)}

For the objective assessment of the stroboscopic effect, the stroboscopic effect visibility measure (symbol $M \mathrm{vs}$ ) is available. The measurement duration is typically one second, in accordance with the recommendations in CIE TN 006:2016 (CIE 2016). The measurement method for the stroboscopic effect of lighting equipment is described in IEC TR 63158:2018 (IEC 2018). 


\subsubsection{Perceptual modulation}

The purpose of the quantity called perceptual modulation (symbol $M \mathrm{P}$ ) is to accurately predict the human flicker perception for any lamp, light output waveform and frequency. Specifically, this quantity deals with the direct perception of flicker in which the frequency of modulation is low enough to be seen directly (i.e. $<80 \mathrm{~Hz}$ ).

The ASSIST document includes details on measurement equipment and conditional requirements (ASSIST 2015).

\section{Measurement recommendations}

\subsection{General recommendations}

General laboratory requirements and best practices should be observed. When testing specific products, the relevant IEC product performance standard (or relevant local regulation) should be followed as general guidance when operating the device under test (DUT).

For LED products covered under CIE S 025/E:2015 (CIE 2015), it is good practice that ambient conditions and operation instructions of the DUT as prescribed in that standard should be observed. Whenever a reference is made to the 'relevant' test standard in the following clause, for LED products this means CIE S 025/E:2015 (CIE 2015). For other products, this would be the relevant IEC product standard.

For the correct calculation of TLM and TLA quantities either the waveform (time domain data) or the power spectral density (frequency domain data) of the waveform is required. The relation between the time domain and the frequency domain is the Fourier (or inverse Fourier) transformation. This TN will focus on the time domain measurement including quantities and quantity-specific recommendations. Further details on the calculation of the Power Spectral Density (PSD) using a Fast Fourier Transform (FFT) are subject of a Technical Report, which is in preparation by CIE TC 2-89 and will be published at a later stage.

NOTE Stroboscopic visibility measure (SVM) is one of the quantities requiring calculation of the PSD using an FFT (see also 4.4.3).

\subsection{Field measurements}

A field measurement is considered to be any measurement that is not done under controlled laboratory conditions, e.g. a measurement in an office or outdoors. For the purpose of this document, any measurement not performed under standard test conditions is a field measurement, the standard conditions of which are described in product performance standards or measurement standards (e.g. CIE S 025/E:2015 (CIE 2015)).

Field measurements can be considerably different from laboratory measurements. If conditions are not as well controlled, the measurement can be used to determine the performance of the DUT under 'less controlled' conditions or in a specific application. A measurement done under mainly uncontrolled conditions is only suited for determining the TLM or TLA aspect of that specific environment, and not of a specific device.

Laboratory measurement results and field measurement results should not be compared directly. Nor should laboratory measurements be assumed to be directly applicable in field conditions.

When field measurements are reported (or results discussed), the conditions under which the measurements were performed should be listed, as far as possible similar to how laboratory measurements are reported. In particular a statement that the measurements were performed as a 'field measurement' should be noted. 
For any field measurements as much detail as is known should be listed in a measurement report. Even a simple statement can suffice (e.g. "connected to mains", or "daylight present"). If no information is available, this should be mentioned as well. The following list describes factors that can significantly influence field measurements:

- power supply characteristics, e.g. AC voltage waveform;

- settings of lighting controls (dimmers, pulse-width modulation (PWM) settings, colour setting for tuneable lights);

- ambient temperature of the DUT;

- static background illumination from other sources, including daylight and other sources;

- non-static background illumination from other TLM sources;

- mechanical vibrations strong enough to influence the measurement.

\subsection{Recommendations for acquiring the waveform}

\subsubsection{General}

TLA metrics are usually defined with regards to light modulation within a specific frequency band, whereas TLM and corresponding metrics may go beyond those specified frequency bands.

The knowledge of the source's spectrum, as well as the modulation frequency, might limit the minimum requirements for acquiring the light waveform. In case that one or more parameters are unknown, it is recommended to use more stringent equipment recommendations (e.g. starting from high sampling frequency and sampling time and reducing accordingly).

Recommendation for sampling frequency: $20 \mathrm{kHz}$ (or higher).

Recommendation for sampling time: $1 \mathrm{~s}$ (or longer).

Recommendation for spectral responsivity: spectral luminous efficiency function for photopic vision $V(\lambda)$.

Beside these general recommendations, some metrics with specific recommendations can be found in 4.4 .

\subsubsection{Sampling}

The Nyquist theorem states that a signal should be sampled with twice the highest frequency present to accurately be able to reconstruct the actual (non-sampled) signal. This is the case for ideal signals and ideal equipment. As this is only a theoretical case, the general recommendation is to oversample by a factor of 10 (instead of 2).

When considering a Fourier Transform or the PSD of the waveform, the total length of the sampled waveform determines the frequency resolution of the calculated PSD. To accurately determine metrics relying on the calculation of the PSD, a minimum sampling time is recommended.

NOTE A high sampling rate and long sampling time means a large sample size.

\subsubsection{Linearity}

The linearity of the measurement system should be characterized and non-linearities should either be corrected for or taken into account in the measurement uncertainties. For more information see ISO/CIE 19476:2014 (ISO/CIE 2014) and CIE 231:2019 (CIE 2019).

NOTE A detector is usually linear only over a certain range of input levels. Outside this range it becomes non-linear. The linearity range should be stated when reporting linearity. 


\subsubsection{Signal-to-noise ratio}

The signal-to-noise ratio (SNR) of the full measurement system should be considered. It needs to be large enough so that measurements of fluctuations in the signal are not significantly affected by noise. Some types of noise can be reduced by taking averages of repeat measurements.

There are several factors affecting the SNR:

- amplitude range and resolution of the digitizer or oscilloscope;

- electrical noise from any part of the equipment;

- level of the background light;

- responsivity of the photodetector.

Every factor should be understood, as some might directly affect the signal, while others might affect the noise levels. The intention is to keep the SNR as high as possible.

\subsection{Quantity-specific recommendations}

\subsubsection{TLM quantities (general)}

In any specific measurement situation, the minimum requirements to adequately measure a TLM quantitiy will depend on knowledge of the signal. If there is only a mains frequency component, measurement conditions are less strict compared to when the signal is a $1 \mathrm{kHz}$ or higher modulation.

\subsubsection{Short-term flicker index}

The sampling time required to determine the short-term flicker index correctly is longer than for most other metrics. However, a slightly lower sampling frequency suffices. These requirements are due to the low frequency $(<80 \mathrm{~Hz})$ effects that this index describes.

IEC TR 61547-1:2020 (IEC 2020) specifically mentions measurements of illuminance, however the short-term flicker index can be calculated on any luminous quantity measured with appropriate equipment.

Recommendation for sampling frequency: $10 \mathrm{kHz}$ (or higher).

Recommendation for sampling time: $60 \mathrm{~s}$ (or longer). See Note 2 for further details.

See IEC TR 61547-1:2020 (IEC 2020) for additional measurement recommendations.

NOTE 1 IEC TR 61547-1:2020 (IEC 2020) recommends a specific setup and equipment combination, which has more stringent requirements. When that measurement method is followed, the recommendations from this TN are superseded by the specific requirements in the IEC TR 61547-1:2020.

NOTE 2 The calculation of the short-term flicker index includes application of a filter with a longer response time to the measured data. This leads to the need for additional $60 \mathrm{~s}$ of acquisition to allow for the settling time of the filter.

\subsubsection{Stroboscopic visibility measure}

The stroboscopic effect from periodic waveforms can be seen up to frequencies of $2,5 \mathrm{kHz}$. Even though stroboscopic effects are visible below $80 \mathrm{~Hz}$, the most noticeable effect in that frequency range is flicker. For this reason, the minimum recommended sampling frequency is $20 \mathrm{kHz}$ with a sample duration of one second (minimum) to be able to verify the periodicity of 
the waveform. The sample duration should be such that the sample has an integer number of periods of the waveform.

See IEC TR 63158:2018(E) (IEC 2018) for additional measurement recommendations.

\subsubsection{Perceptual modulation}

See 'Recommended metric for assessing the direct perception of light source flicker, volume 11, issue 3:2015' (ASSIST 2015) for additional measurement recommendations.

\subsection{Measurement equipment}

\subsubsection{General}

The measurement equipment should be selected to meet desired sampling, accuracy, detection limits, repeatability, reproducibility and robustness. The equipment selection is always a balance between technical requirements, risks, and economic considerations.

There might be particular standards or regulations that set requirements different from what is recommended in this document. As long as these requirements are stricter than what is listed in this document, it is recommended to maintain the requirements from those documents as there might be specific reasoning behind them which are not considered in the generalized recommendations in this Technical Note.

The recommendations made in this subclause typically relate to laboratory measurement and not field measurement.

\subsubsection{Measured quantities and measurement geometries}

Waveform data can be recorded using many different input optics. Input optics are categorized into illuminance optics and luminance optics. A few examples of additional instruments to be combined with the input optics include:

- integrating sphere and photometer (or photodiode for non-visible radiation applications);

- optical bench and illuminance meter;

- optical bench and luminance meter;

- goniophotometer with fast, time-resolved measurements;

- illuminance meter with fast, time-resolved measurements (in field measurements);

An example of an integrating-sphere setup is given in Clause 6.

The specific measurement geometry and input optic used is dependent on the specific application and should be described in the final measurement report or when discussing the results.

Whichever input optic or geometry is chosen, the relevant standards (if applicable) should be referred to for additional requirements, e.g.:

- illuminance meter: ISO/CIE 19476:2014 (ISO/CIE 2014);

- integrating sphere: CIE 084:1989 (CIE 1989) and CIE S 025/E:2015 (CIE 2015);

- goniophotometer: CIE 121:1996 (CIE 1996) and CIE S 025/E:2015 (CIE 2015);

- luminance meter: ISO/CIE 19476:2014 (ISO/CIE 2014). 


\subsubsection{Electrical supply and measurement}

\subsubsection{General}

This document does not give recommendations about the measurement of electrical parameters. However, it is recommended to use equipment that fulfils requirements given in relevant test standards or regulations.

The power supply can have a significant effect on the time-modulated output of the DUT and so will ideally fulfil the requirements as set out in the relevant test standards. Following these requirements should minimize any additional effects introduced by the power supply.

\subsubsection{Dimmer}

Adding a dimmer to the system will in almost all cases influence the results, even when it is set to $100 \%$ output or 'no-dimming' mode. There are several different dimmer types and even within the same dimmer type, the response of the DUT to the dimmer might differ.

If the purpose of the test is to only test the DUT, the dimmer should be taken out of the electrical supply system or bypassed completely. If a dimmer is used, the type, model and manufacturer should be reported with the results.

Different regional standards may require testing of a light source with and without dimmers. In these cases, it is important to follow the requirements stated in those standards.

The use of standardized dimmers, like the dimmer specified in NEMA SSL 7A-2015 (NEMA 2015), should be considered when making comparisons between different measurements (e.g. when comparing results from different laboratories on the same DUT).

\subsubsection{Test chamber}

For laboratory measurements other than in an integrating sphere, it is important to use a room or test chamber where any ambient light is minimized.

Other environmental conditions can also impact the measurement results and should be considered. CIE S 025/E:2015 (CIE 2015), subclause 4.2, lists some of the most important conditions to consider, and in subclause 4.1.1 of the same document several additional conditions are listed which are known to influence measurement results.

When reporting results, the optical input method (e.g. integrating sphere, dark room with optical bench and illuminance meter, goniophotometer laboratory) should be listed with as much detail as possible.

\subsubsection{Photodetector}

\subsubsection{General}

All quantities and measurements in this document relate to human perception of the modulated system, hence it is necessary to use a $V(\lambda)$-corrected detector.

The detector does not require an absolute calibration, as the quantities in this document are independent of absolute levels.

The frequency response of the detector should sufficiently cover the frequency range of the sampling given in the recommendations for the desired quantity. However, if a transimpedance amplifier is also used (see 4.5.5.2), then the combined frequency response should be considered. 


\subsubsection{Transimpedance amplifier}

Photodiodes are usually connected to a transimpedance amplifier to provide a linear response and a voltage output signal. This can be integrated into the detector but can be a separate piece of equipment.

The gain of the amplifier should be set, so that the output matches the range of the digitizer.

\subsubsection{Dark correction}

Part of the ability of a linear detection system is a zero-reading when there is no input to the measurement system. This means that when there is no light coming to the detector, it should be reading ' 0 ', or close to 0 . This can be verified with the system in a dark room and any lights off. If in this case, the detector is reading a non-zero value, the measurement system should be adjusted so it is reading a zero value. How this is done depends on the specific equipment. Some devices have an amplification offset adjustment, a 'zero-level' adjustment option, or the offset level may be subtracted in software. The documentation of the equipment should be referred to with respect to the specifications of this correction measurement.

In case the equipment does not allow respective zero adjustments, it is usually possible to perform subtraction of DC offsets manually after having recorded the waveform (but before any additional calculations).

\subsection{6 (Low-pass) filter}

Depending on the frequency requirements of the metric being measured, it may be helpful to filter out higher frequency components. The cut-off frequency is determined by the required frequency range in the calculation of the desired metric.

Attention should be paid when using filters as they can strongly impact the linearity of the system. Only Filters with a flat response in the desired frequency range should be used.

\subsubsection{Oscilloscope or digitizer}

The analogue output of the amplifier needs to be converted to a digital signal. This is often done using an oscilloscope or digital multimeter, but it is not limited to these. Any digitizer technology can be used if it can fulfil all mentioned recommendations.

For the selection of a digitizer, the vertical resolution (usually specified in bits) is an important property to consider as it has a direct effect on the amplitude resolution. An 8-bit system would normally not have enough resolution and at least 10 bits and preferably 12 bits is recommended.

\section{Measurement evaluation}

\subsection{General}

Once the time domain signal has been digitized and recorded, it can be evaluated. Depending on the specific metric, this will involve different calculations. Further frequency filtering might be needed, and some metrics require the PSD to be calculated.

Several mathematical tools are available for these calculations, however the usage and description of these is beyond the scope of this document. 


\subsection{Calculation of the PSD}

A Discrete Fourier Transform (DFT) must be applied to the time domain signal to determine the PSD, usually implemented using an FFT algorithm. These calculations are not trivial and can easily cause significant errors in the final measurement results.

\section{Measurement setup example}

An example measurement set-up for laboratory measurements of TLM and TLA quantities will have a stable power source to power the DUT. It might contain a dimmer, or several dimmers, but this is optional and depends on the requirements of the person requesting the test or of a particular regulation.

The DUT is located in a test chamber or integrating sphere that eliminates stray light from other possible light sources. A photodetector gathers the analogue optical signal from the source and outputs a photocurrent. A transimpedance amplifier converts the photocurrent to a voltage signal. Impedance matching of the photodetector, transimpedance amplifier and voltage capture device used is important to ensure correct measurements. The frequency response of the full system must be validated for the bandwidth required for the quantity of interest. A low-pass filter may be utilized to eliminate higher frequency components that are not utilized in any calculations. The analogue signal is digitized and converted to a digital data file where calculations for the metrics are then made. Figure 1 shows an example of such a setup.

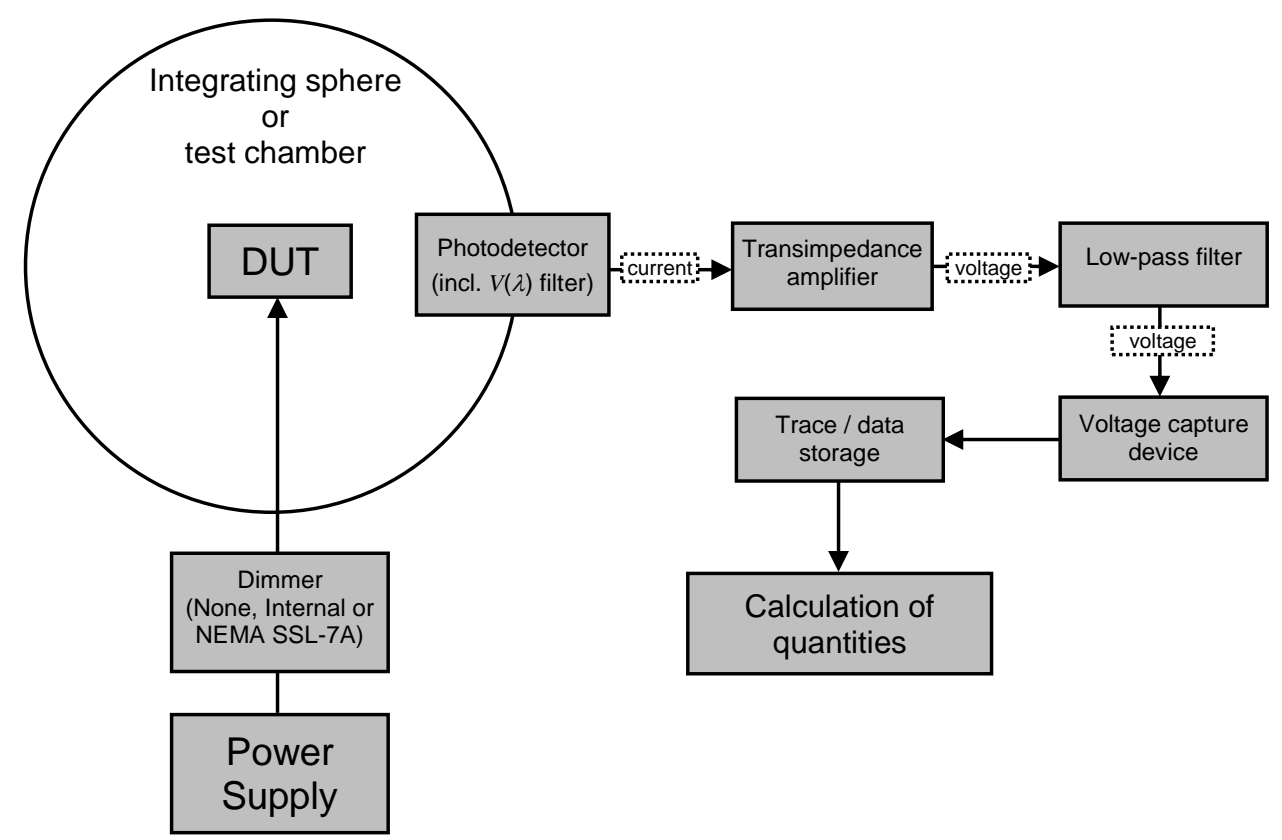

Figure 1 - Example of a laboratory setup used to measure TLM and TLA properties of a DUT

\section{Reporting of results and measurement uncertainty}

When reporting the results of any measurement, an evaluation of the measurement uncertainty should be included. It is understood that at the time of the writing of this document, the calculation of measurement uncertainty for temporal light modulation is not fully understood and many academic and practical research studies are looking into this. There are many factors influencing the results and some are not easy to analyse. 
When a full uncertainty study is not done, it is extremely important to list full details of measurement equipment and equipment settings used for the measurements and environment conditions at the time of the measurement.

Currently a standardized file format for storing a waveform has not been set. This document proposes to store the data in a comma-separated values (csv) file, with the following formatting:

- the first line of the file containing the resolution of the time axis (in seconds)

- the second line containing the total number of amplitude data points

- after this every line containing a single amplitude-normalized data point

A normalized data point is recommended as that means waveforms are easily comparable and interchangeable between different calculation algorithms. Normalization should be to the average value of the complete waveform data as shown in Equation (2).

$$
y_{\text {norm }}=\frac{y}{\langle y\rangle}
$$

where

$y_{\text {norm }}$ is the normalized data point;

$y \quad$ is the data point in the waveform;

$\langle y\rangle$ is the average of all data points in the waveform dataset.

\section{References}

ASSIST, 2015. Recommended metric for assessing the direct perception of light source flicker. 11(3).

CIE, 1989. CIE 084-1989. Measurement of Luminous Flux. Vienna: CIE.

CIE, 2015. CIE S 025/E:2015. Test Method for LED Lamps, LED Luminaires and LED Modules. Vienna: CIE.

CIE, 2016. CIE TN 006:2016. Visual Aspects of Time Modulated Lighting Systems Definitions and Measurement Models. Vienna: CIE.

CIE, 2020. CIE S 017:2020. ILV: International Lighting Vocabulary 2nd Edition. Vienna: CIE.

IEC, 1988. IEC 60050-701:1988. International Electrotechnical Vocabulary (IEV) - Part 701: Telecommunications, channels and networks. Geneva: IEC.

IEC, 1998. IEC 60050-713:1998. International Electrotechnical Vocabulary (IEV) - Part 713: Radiocommunications: transmitters, receivers, networks and operation. Geneva: IEC.

IEC, 2009. IEC 60050-103:2009. International Electrotechnical Vocabulary (IEV) - Part 103: Mathematics - Functions. Geneva: IEC.

IEC, 2020. IEC TR 61547-1:2020(E). Equipment for general lighting purposes - EMC immunity requirements - Part 1: An objective light flickermeter and voltage fluctuation immunity test method. Geneva: IEC.

IEC, 2018. IEC TR 63158:2018(E). Equipment for general lighting purposes - Objective test method for stroboscopic effects of lighting equipment. Geneva: IEC.

ISO/CIE, 2014. ISO/CIE 19476:2014(E). Characterization of the performance of illuminance meters and luminance meters. Vienna: CIE.

NEMA, 2015. SSL 7A-2015. Phase-Cut Dimming for Solid State Lighting: Basic Compatibility. Virginia: NEMA. 